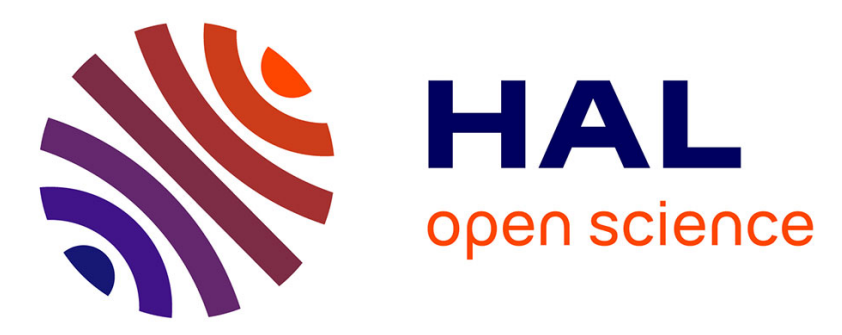

\title{
Optimal Kinematic Redundancy Planning for Planar Mobile Cable-Driven Parallel Robots
}

\author{
Tahir Rasheed, Philip Long, David Marquez-Gamez, Stéphane Caro
}

\section{To cite this version:}

Tahir Rasheed, Philip Long, David Marquez-Gamez, Stéphane Caro. Optimal Kinematic Redundancy Planning for Planar Mobile Cable-Driven Parallel Robots. The ASME 2018 International Design Engineering Technical Conferences \& Computers and Information in Engineering Conference IDETC/CIE 2018, Aug 2018, Quebec city, Canada. 10.1115/DETC2018-86182 . hal-01863727

\section{HAL Id: hal-01863727 https://hal.science/hal-01863727}

Submitted on 29 Aug 2018

HAL is a multi-disciplinary open access archive for the deposit and dissemination of scientific research documents, whether they are published or not. The documents may come from teaching and research institutions in France or abroad, or from public or private research centers.
L'archive ouverte pluridisciplinaire HAL, est destinée au dépôt et à la diffusion de documents scientifiques de niveau recherche, publiés ou non, émanant des établissements d'enseignement et de recherche français ou étrangers, des laboratoires publics ou privés. 


\section{The ASME 2018 International Design Engineering Technical Conferences \& Computers and Information in Engineering Conference IDETC/CIE 2018}

August 26-292018Quebec CityCanada

\section{DETC2018-86182}

\section{OPTIMAL KINEMATIC REDUNDANCY PLANNING FOR PLANAR MOBILE CABLE-DRIVEN PARALLEL ROBOTS}

\author{
Tahir Rasheed \\ École Centrale de Nantes \\ Laboratoire des Sciences du Numérique de Nantes, \\ Tahir.Rasheed@Is2n.fr

\section{David Marquez-Gamez} \\ IRT Jules Verne, Chemin du Chaffault, \\ 44340, Bouguenais, France, \\ david.marquez-gamez@irt-jules-verne.fr
} UMR CNRS 6004 1, rue de la Noë, 44321 Nantes, France Northeastern University, Boston, Massachusetts, USA

\author{
Philip Long \\ RIVeR Lab, Department of \\ electrical and computing engineering, \\ p.long@northeastern.edu
}

\author{
Stéphane Caro* \\ CNRS, Laboratoire des Sciences du Numérique de Nantes, \\ UMR CNRS 6004, 1, rue de la Noë, 44321 Nantes, France \\ stephane.caro@Is2n.fr
}

\begin{abstract}
Mobile Cable-Driven Parallel Robots (MCDPRs) are special type of Reconfigurable Cable Driven Parallel Robots (RCDPRs) with the ability of undergoing an autonomous change in their geometric architecture. MCDPRs consists of a classical Cable-Driven Parallel Robot (CDPR) carried by multiple Mobile Bases (MBs). Generally MCDPRs are kinematically redundant due to the additional mobilities generated by the motion of the MBs. As a consequence, this paper introduces a methodology that aims to determine the best kinematic redundancy scheme of Planar MCDPRs (PMCDPRs) with one degree of kinematic redundancy for pick-and-place operations. This paper also discusses the Static Equilibrium (SE) constraints of the PMCDPR $M B$ s that are needed to be respected during the task. A case study of a PMCDPR with two MBs, four cables and a three degree-offreedom (DoF) Moving Platform (MP) is considered.
\end{abstract}

\section{INTRODUCTION}

Cable-Driven Parallel Robots (CDPRs) are a particular type of parallel manipulators with cables instead of rigid links. The

*Address all correspondence to this author. platform motion is generated by changing the cable lengths between the Moving Platform (MP) and the fixed base frame. CDPRs are used for several applications that requires high accelerations [1], large payload capabilities [2], and large workspace [3].

One of the major drawbacks in classical CDPRs with fixed cable layout, i.e. fixed exit points and cable configuration, is the potential collisions between the cables and the surrounding environment. These collisions significantly reduce the CDPR workspace and the platform stiffness. A proposed solution is Reconfigurable Cable-Driven Parallel Robots (RCDPRs) whose geometric architecture can be altered to achieve better performances, e.g. lower cable tensions, larger workspace and increasing platform stiffness [4]. However, reconfigurability is a manual, discrete and a time consuming task.

In [5] a novel concept of Mobile Cable-Driven Parallel Robots (MCDPRs) using a combination of Mobile Bases (MBs) and a CDPR in order to achieve autonomous reconfigurability of RCDPRs is presented. A MCDPR is composed of a classical CDPR with $q$ cables and a $n$ degree-of-freedom (DoF) MP mounted on $p$ MBs [5]. In our previous work [6] we determine the Available Wrench Set (AWS) for Planar MCDPRs (PMCD- 


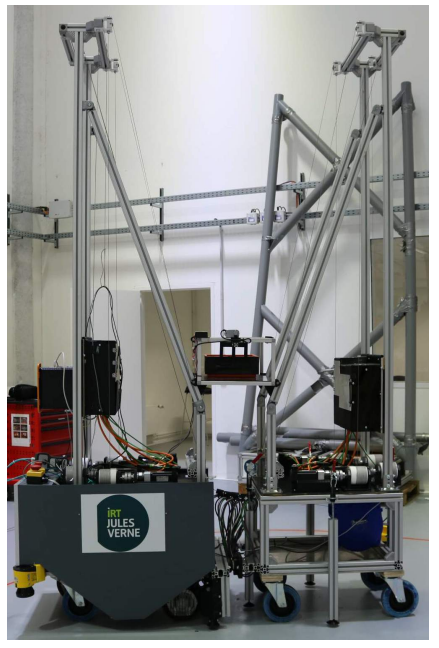

a

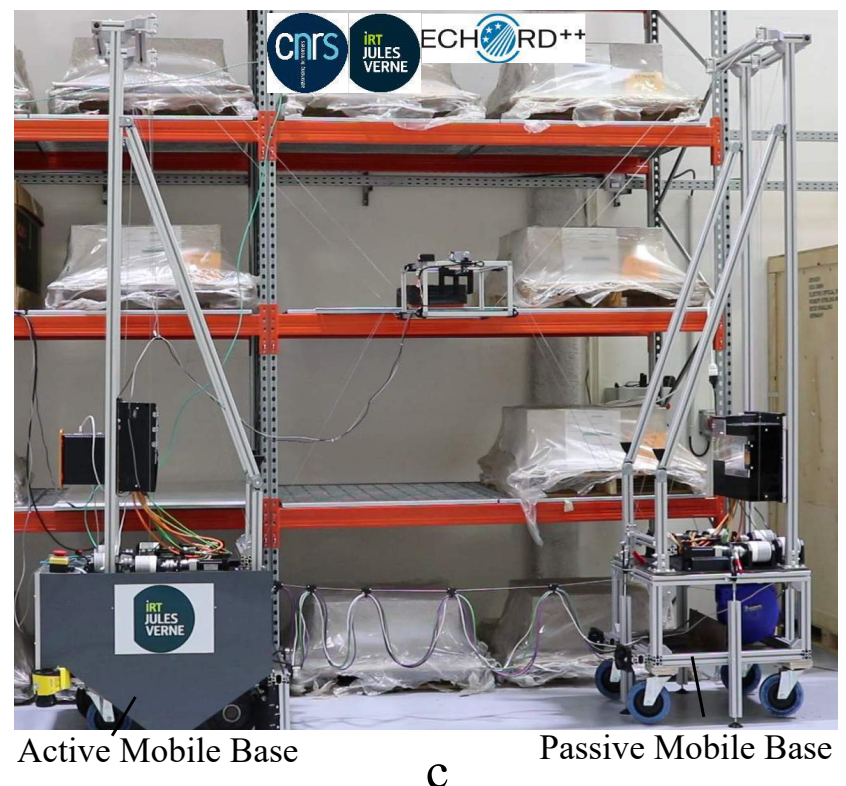

C

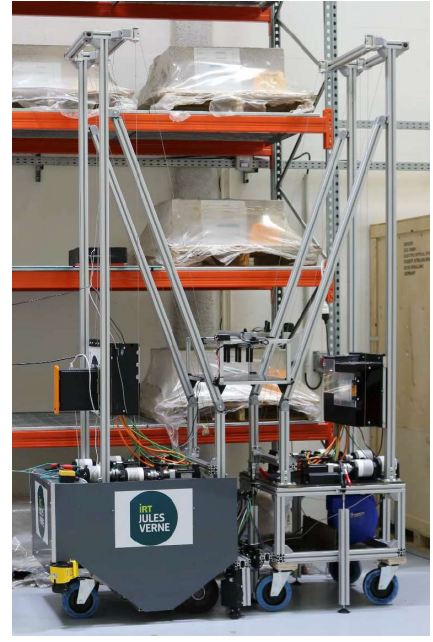

b 
where $\tau_{i j}$ is the tension in the $i$ th cable mounted on $\mathscr{M}_{j}$ bounded between minimum $\underline{\tau}_{i j}$ and maximum $\bar{\tau}_{i j}$ cable tension limits.

Cables are assumed to be straight and massless, thus can be modeled as a Revolute-Prismatic-Revolute (RPR) kinematic chain. For the MCDPR under study, the MBs are capable of generating a single DoF translational motion along $x_{0}$. In this paper, $\mathscr{M}_{1}$ is considered as passive while $\mathscr{M}_{2}$ is considered as active mobile base. The position of $\mathscr{M}_{1}\left(\mathscr{M}_{2}\right.$, resp.) along $x_{0}$ is defined by $\rho_{1}$ ( $\rho_{2}$ resp.) with respect to the frame $\mathscr{F}_{1}\left(\mathscr{F}_{2}\right.$, resp.) attached to it. The twist capability of $\mathscr{M}_{2}$ is quasi-static and negligible, thus only the SE of the MBs in taken into account without considering the dynamics of the latter.

\section{WRENCH FEASIBILITY}

For MCDPRs, the Available Wrench Set (AWS) is defined as the set of wrenches a mechanism can generate while respecting the cable tension limits and the SE constraints of the MBs [6]. The approach proposed in [6] characterizes the AWS of the MCDPR where the end effector is treated as a point mass. In this section we extend the formulation to consider a moving platform subject to planar forces and moment.

\subsection{Static Equilibrium of the manipulator}

The Static Equilibrium (SE) of the MP is expressed as [8,9]:

$$
\mathbf{W} \boldsymbol{\tau}+\left[\begin{array}{c}
\mathbf{f}_{e} \\
m_{e}
\end{array}\right]=\mathbf{0}_{3}
$$

where $\mathbf{W}$ is a $(n \times q)$ wrench matrix mapping the cable tension vector $\tau \in \mathbb{R}^{q}$ onto the wrenches generated by the cables on the end-effector. $\mathbf{f}_{e}=\left[\begin{array}{ll}f_{e}^{x} & f_{e}^{y}\end{array}\right]^{T}$ and $m_{e}$ respectively denote the external forces and moment applied by the MP. For the MCDPR under study in Fig. 2, Eqn. (2) is expressed as:

$\left[\begin{array}{cccc}\mathbf{u}_{11} & \mathbf{u}_{21} & \mathbf{u}_{12} & \mathbf{u}_{22} \\ \mathbf{r}_{11}^{T} \mathbf{E}^{T} \mathbf{u}_{11} & \mathbf{r}_{21}^{T} \mathbf{E}^{T} \mathbf{u}_{21} & \mathbf{r}_{12}^{T} \mathbf{E}^{T} \mathbf{u}_{12} & \mathbf{r}_{22}^{T} \mathbf{E}^{T} \mathbf{u}_{22}\end{array}\right]\left[\begin{array}{c}\tau_{11} \\ \tau_{21} \\ \tau_{12} \\ \tau_{22}\end{array}\right]+\left[\begin{array}{c}\mathbf{f}_{e} \\ m_{e}\end{array}\right]=\mathbf{0}_{3}$

with

$$
\mathbf{E}=\left[\begin{array}{cc}
0 & -1 \\
1 & 0
\end{array}\right]
$$

where $\mathbf{r}_{i j}$ is a vector pointing from the reference point $(P)$ of the MP to the cable attachment point $B_{i j}$. From the free body diagram in Fig. 2, the Static Equilibrium of $\mathscr{M}_{j}$ can be expressed

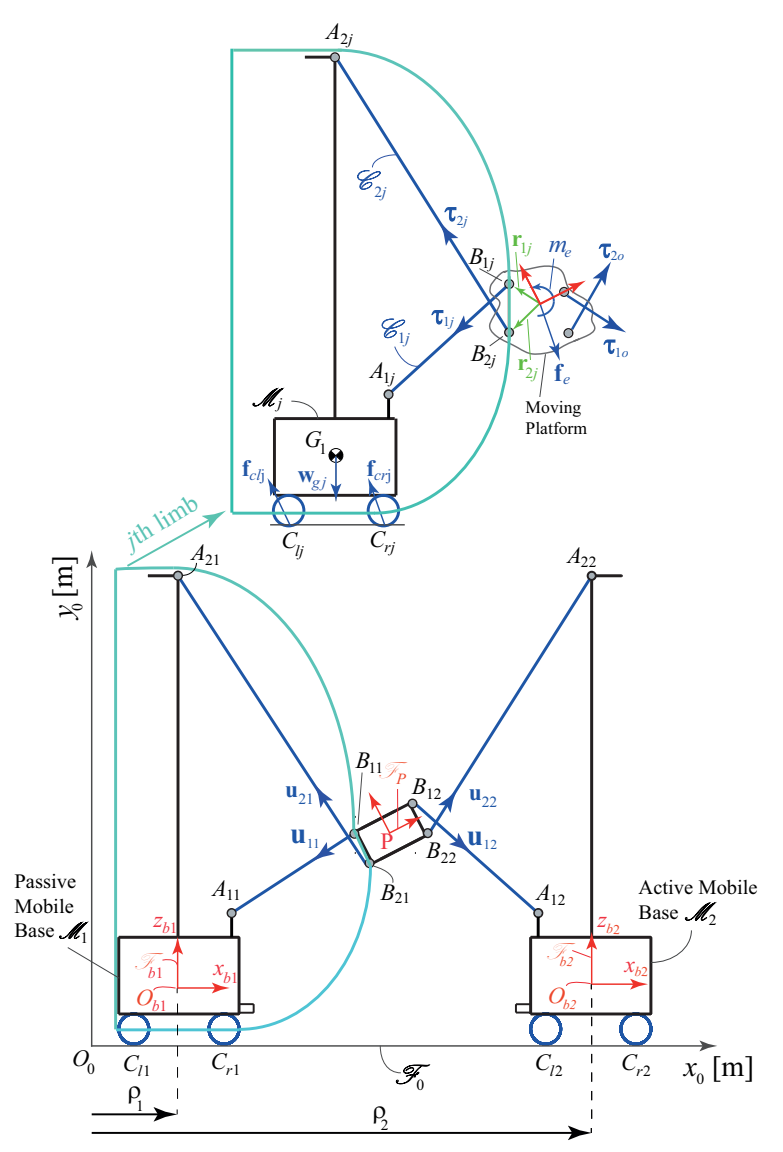

FIGURE 2. PARAMETERIZATION OF PLANAR FASTKIT

as:

$$
\mathbf{w}_{g j}-\sum_{i=1}^{2} \boldsymbol{\tau}_{i j}+\mathbf{f}_{c l j}+\mathbf{f}_{c r j}=\mathbf{0}_{2}
$$

$$
m_{O j}=\mathbf{g}_{j}^{T} \mathbf{E}^{T} \mathbf{w}_{g j}-\sum_{i=1}^{2} \mathbf{b}_{i j}^{T} \mathbf{E}^{T} \boldsymbol{\tau}_{i j}+\mathbf{c}_{l j}^{T} \mathbf{E}^{T} \mathbf{f}_{c l j}+\mathbf{c}_{r j}^{T} \mathbf{E}^{T} \mathbf{f}_{c r j}=0
$$

where $m_{O j}$ denotes the moment of $\mathscr{M}_{j}$ about point $O_{0}$. The weight vector of $\mathscr{M}_{j}$ is denoted by $\mathbf{w}_{g j} . \quad \mathbf{f}_{c l j}=\left[\begin{array}{ll}f_{c l j}^{x} & f_{c l j}^{y}\end{array}\right]^{T}$ and $\mathbf{f}_{c r j}=\left[\begin{array}{ll}f_{c r j}^{x} & f_{c r j}^{y}\end{array}\right]^{T}$ denotes the reaction forces from the ground on the left and right contact points $C_{l j}$ and $C_{r j}$ of $\mathscr{M}_{j}$. $\mathbf{b}_{i j}=\left[\begin{array}{ll}b_{i j}^{x} & b_{i j}^{y}\end{array}\right]^{T}, \mathbf{c}_{l j}=\left[\begin{array}{ll}c_{l j}^{x} & c_{l j}^{y}\end{array}\right]^{T}$ and $\mathbf{c}_{r j}=\left[\begin{array}{ll}c_{r j}^{x} & c_{r j}^{y}\end{array}\right]^{T}$ denote the Cartesian coordinate vectors of points $B_{i j}, C_{l j}$ and $C_{r j}$, respectively. $\mathbf{g}_{j}=\left[\begin{array}{ll}g_{j}^{x} & g_{j}^{y}\end{array}\right]^{T}$ is the Cartesian coordinate vector of the center of gravity $G_{j}$. Note that the superscripts $x$ and $y$ in the previous vectors denotes their $x$ and $y$ components.

Let $m_{C r j}$ represent a moment generated at the right contact point $C_{r j}$ when $\mathscr{M}_{j}$ loses the ground contact at point $C_{l j}$, i.e. $m_{C r j}$ 
is expressed as:

$$
m_{C r j}=\left(\mathbf{g}_{j}-\mathbf{c}_{r j}\right)^{T} \mathbf{E}^{T} \mathbf{w}_{g j}+\sum_{i=1}^{2}\left(\mathbf{c}_{r j}-\mathbf{b}_{i j}\right)^{T} \mathbf{E}^{T} \tau_{i j}
$$

The vector sum between the points $C_{r j}, B_{i j}$ and $P$ is expressed as:

$$
\left(\mathbf{c}_{r j}-\mathbf{b}_{i j}\right)+\mathbf{r}_{i j}+\left(\mathbf{p}-\mathbf{c}_{r j}\right)=\mathbf{0}
$$

Thus,

$$
\left(\mathbf{c}_{r j}-\mathbf{b}_{i j}\right)=-\mathbf{r}_{i j}-\left(\mathbf{p}-\mathbf{c}_{r j}\right)
$$

Substituting Eqn. (9) in Eqn. (7) yields:

$$
m_{C r j}=\left(\mathbf{g}_{j}-\mathbf{c}_{r j}\right)^{T} \mathbf{E}^{T} \mathbf{w}_{g j}-\sum_{i=1}^{2}\left(\mathbf{p}-\mathbf{c}_{r j}\right)^{T} \mathbf{E}^{T} \boldsymbol{\tau}_{i j}-\sum_{i=1}^{2} \mathbf{r}_{i j}^{T} \mathbf{E}^{T} \boldsymbol{\tau}_{i j}
$$

From Eqn. (3):

$$
\begin{gathered}
\sum_{i=1}^{2} \boldsymbol{\tau}_{i j}=\mathbf{f}-\sum_{i=1}^{2} \tau_{i o}, \quad o \neq j \\
\sum_{i=1}^{2} \mathbf{r}_{i j}^{T} \mathbf{E}^{T} \boldsymbol{\tau}_{i j}=m-\sum_{i=1}^{2} \mathbf{r}_{i o}^{T} \mathbf{E}^{T} \tau_{i o}, \quad o \neq j
\end{gathered}
$$

where $\mathbf{f}=\left[\begin{array}{ll}f^{x} & f^{y}\end{array}\right]^{T}$ and $m$ denote the forces and moment generated by the cables onto the MP, namely,

$$
\mathbf{f}=-\mathbf{f}_{e}, \quad m=-m_{e}
$$

Substituting Eqn. (12) in Eqn. (10) yields:

$$
\begin{aligned}
m_{C r j} & =-\left[\left(\mathbf{p}-\mathbf{c}_{r j}\right)^{T} \mathbf{E}^{T} 1\right]\left[\begin{array}{c}
\mathbf{f} \\
m
\end{array}\right]+\left(\mathbf{g}_{j}-\mathbf{c}_{r j}\right)^{T} \mathbf{E}^{T} \mathbf{w}_{g j} \\
& +\sum_{i=1}^{2}\left(\mathbf{p}-\mathbf{c}_{r j}\right)^{T} \mathbf{E}^{T} \boldsymbol{\tau}_{i o}+\sum_{i=1}^{2} \mathbf{r}_{i o}^{T} \mathbf{E}^{T} \boldsymbol{\tau}_{i o}, o \neq j
\end{aligned}
$$

Similarly, the moment $m_{C l j}$ generated at the left contact point $C_{l j}$ takes the form:

$$
\begin{gathered}
m_{C l j}=-\left[\begin{array}{ll}
\left.\left(\mathbf{p}-\mathbf{c}_{l j}\right)\right]^{T} \mathbf{E}^{T} & 1
\end{array}\right]\left[\begin{array}{c}
\mathbf{f} \\
m
\end{array}\right]+\left(\mathbf{g}_{j}-\mathbf{c}_{l j}\right)^{T} \mathbf{E}^{T} \mathbf{w}_{g j} \\
+\sum_{i=1}^{2}\left(\mathbf{p}-\mathbf{c}_{l j}\right)^{T} \mathbf{E}^{T} \boldsymbol{\tau}_{i o}+\sum_{i=1}^{2} \mathbf{r}_{i o}^{T} \mathbf{E}^{T} \boldsymbol{\tau}_{i o}, o \neq j
\end{gathered}
$$

For both the MBs to be in SE, the moments $m_{C r j}\left(m_{C l j}\right.$, resp.) should be always counterclockwise (clockwise, resp.) at contact points $C_{r j}\left(C_{l j}\right.$, resp.), namely,

$$
m_{C r j} \geq 0, \quad m_{C l j} \leq 0, \quad j=1,2
$$

\subsection{Available Wrench Set}

The AWS of PMCDPR with a point mass MP in [6] is extended to define the AWS $(\mathscr{A})$ of the MCDPR under study, expressed as:

$$
\begin{aligned}
\mathscr{A}= & \left\{\left[\begin{array}{c}
\mathbf{f} \\
m
\end{array}\right] \in \mathbb{R}^{3} \mid\left[\begin{array}{c}
\mathbf{f} \\
m
\end{array}\right]=\mathbf{W} \tau, \underline{\tau}_{i j} \leq \tau_{i j} \leq \bar{\tau}_{i j},\right. \\
& \left.m_{C r j} \geq 0, m_{C l j} \leq 0, i=1,2, j=1,2\right\} .
\end{aligned}
$$

The AWS defined in Eqn. (16) corresponds to a $n$ dimensional convex polytope that can be represented as the intersection of the half-spaces bounded by its hyperplanes. It depends on the MCDPR configuration and the constraints associated with the cable tension limits and the SE of the MBs. The facets or hyperplanes of the AWS associated with the cable tension limits can be directly obtained from [6]. However, the SE constraint of the MBs defined by Eqn. (15) are required to be represented in the form of the hyperplanes $[10,11]$.

For completely characterizing the hyperplanes associated with the SE constraints of $\mathscr{M}_{j}$ in $n=3$ dimensional wrench space, the latter must also be independent of $n=3$ number of cable tensions. These $n=3$ cable tensions must consider the cables carried by $\mathscr{M}_{j}$, i.e. $\tau_{1 j}, \tau_{2 j}$ and the combination of $n-m_{j}$ out of $m_{o}, o \neq j$ cables tensions. Thus a single SE constraint will form $C_{n-m_{j}}^{m_{o}}$ number of hyperplanes in $n$-dimensional wrench space. From the SE (Eqn. 3) of the moving platform, the cable tensions $\tau_{1 o}$ and $\tau_{2 o}$ can be expressed as:

$$
\begin{gathered}
-c_{2 j} \mathbf{f}^{T} \mathbf{E}^{T} \mathbf{u}_{1 j}+c_{1 j} \mathbf{f}^{T} \mathbf{E}^{T} \mathbf{u}_{2 j}+c_{2 o} \tau_{2 o} \mathbf{u}_{1 j}^{T} \mathbf{E}^{T} \mathbf{u}_{2 j} \\
\tau_{1 o}=\frac{-m \mathbf{u}_{1 j}^{T} \mathbf{E}^{T} \mathbf{u}_{2 j}+c_{2 j} \tau_{2 o} \mathbf{u}_{2 o}^{T} \mathbf{E}^{T} \mathbf{u}_{1 j}+c_{1 j} \tau_{2 o} \mathbf{u}_{2 j}^{T} \mathbf{E}^{T} \mathbf{u}_{2 o}}{c_{2 j} \mathbf{u}_{1 j}^{T} \mathbf{E}^{T} \mathbf{u}_{1 o}+c_{1 o} \mathbf{u}_{2 j}^{T} \mathbf{E}^{T} \mathbf{u}_{1 j}+c_{1 j} \mathbf{u}_{1 o}^{T} \mathbf{E}^{T} \mathbf{u}_{2 j}}
\end{gathered}
$$

$$
\begin{gathered}
-c_{2 j} \mathbf{f}^{T} \mathbf{E}^{T} \mathbf{u}_{1 j}+c_{1 j} \mathbf{f}^{T} \mathbf{E}^{T} \mathbf{u}_{2 j}+c_{1 o} \tau_{1 o} \mathbf{u}_{1 j}^{T} \mathbf{E}^{T} \mathbf{u}_{2 j} \\
\tau_{2 o}=\frac{-m \mathbf{u}_{1 j}^{T} \mathbf{E}^{T} \mathbf{u}_{2 j}+c_{2 j} \tau_{1 o} \mathbf{u}_{1 o}^{T} \mathbf{E}^{T} \mathbf{u}_{1 j}+c_{1 j} \tau_{1 o} \mathbf{u}_{2 j}^{T} \mathbf{E}^{T} \mathbf{u}_{1 o}}{c_{2 o} \mathbf{u}_{2 j}^{T} \mathbf{E}^{T} \mathbf{u}_{1 j}+c_{2 j} \mathbf{u}_{1 j}^{T} \mathbf{E}^{T} \mathbf{u}_{2 o}+c_{1 i} \mathbf{u}_{2 o}^{T} \mathbf{E}^{T} \mathbf{u}_{2 j}}
\end{gathered}
$$


where

$$
c_{i j}=\mathbf{r}_{i j}^{T} \mathbf{E}^{T} \mathbf{u}_{i j}, \quad i=1,2, \quad j=1,2
$$

By substituting Eqns. (17) and (18) separately in Eqn. (13), a single SE constraint at the contact point $C_{r j}$ will form two hyperplanes in the wrench space expressed as:

$$
\begin{gathered}
\frac{\left[\left(\left(\mathbf{p}-\mathbf{c}_{r j}\right)^{T}-\frac{n_{r j 1}}{d_{r j 1}}\left(c_{2 j} \mathbf{u}_{1 j}^{T}-c_{1 j} \mathbf{u}_{2 j}^{T}\right)\right) \mathbf{E}^{T} 1+\frac{n_{r j 1}}{d_{r j 1}}\left(\mathbf{u}_{1 j}^{T} \mathbf{E}^{T} \mathbf{u}_{2 j}\right)\right]}{\left\|\left(\left(\mathbf{p}-\mathbf{c}_{r j}\right)^{T}-\frac{n_{r j 1}}{d_{r j 1}}\left(c_{2 j} \mathbf{u}_{1 j}^{T}-c_{1 j} \mathbf{u}_{2 j}^{T}\right)\right) \mathbf{E}^{T} 1+\frac{n_{r j 1}}{d_{r j 1}}\left(\mathbf{u}_{1 j}^{T} \mathbf{E}^{T} \mathbf{u}_{2 j}\right)\right\|}\left[\begin{array}{c}
\mathbf{f} \\
m
\end{array}\right] \\
\left(\mathbf{g}_{j}-\mathbf{c}_{r j}\right)^{T} \mathbf{E}^{T} \mathbf{w}_{g j}+\left(\left(\mathbf{p}-\mathbf{c}_{r j}\right)^{T} \mathbf{E}^{T} \mathbf{u}_{2 o}+\mathbf{r}_{2 o}^{T} \mathbf{E}^{T} \mathbf{u}_{2 o}\right) \tau_{2 o} \\
\leq \frac{n_{r j 1}}{d_{r j 1}}\left(c_{2 o} \mathbf{u}_{1 j}^{T} \mathbf{E}^{T} \mathbf{u}_{2 j}+c_{1 j} \mathbf{u}_{2 o}^{T} \mathbf{E}^{T} \mathbf{u}_{2 j}+c_{2 j} \mathbf{u}_{2 o}^{T} \mathbf{E}^{T} \mathbf{u}_{1 j}\right) \tau_{2 o} \\
\left\|\left(\left(\mathbf{p}-\mathbf{c}_{r j}\right)^{T}-\frac{n_{r j 1}}{d_{r j 1}}\left(c_{2 j} \mathbf{u}_{1 j}^{T}-c_{1 j} \mathbf{u}_{2 j}^{T}\right)\right) \mathbf{E}^{T} 1+\frac{n_{r j 1}}{d_{r j 1}}\left(\mathbf{u}_{1 j}^{T} \mathbf{E}^{T} \mathbf{u}_{2 j}\right)\right\|
\end{gathered}
$$

$$
\begin{gathered}
\frac{\left[\left(\left(\mathbf{p}-\mathbf{c}_{r j}\right)^{T}-\frac{n_{r j 2}}{d_{r j 2}}\left(c_{2 j} \mathbf{u}_{1 j}^{T}-c_{1 j} \mathbf{u}_{2 j}^{T}\right)\right) \mathbf{E}^{T} 1+\frac{n_{r j 2}}{d_{r j 2}}\left(\mathbf{u}_{1 j}^{T} \mathbf{E}^{T} \mathbf{u}_{2 j}\right)\right]}{\left\|\left(\left(\mathbf{p}-\mathbf{c}_{r j}\right)^{T}-\frac{n_{r j 2}}{d_{r j 2}}\left(c_{2 j} \mathbf{u}_{1 j}^{T}-c_{1 j} \mathbf{u}_{2 j}^{T}\right)\right) \mathbf{E}^{T} 1+\frac{n_{r j 1}}{d_{r j 1}}\left(\mathbf{u}_{1 j}^{T} \mathbf{E}^{T} \mathbf{u}_{2 j}\right)\right\|}\left[\begin{array}{c}
\mathbf{f} \\
m
\end{array}\right] \\
\left(\mathbf{g}_{j}-\mathbf{c}_{r j}\right)^{T} \mathbf{E}^{T} \mathbf{w}_{g j}+\left(\left(\mathbf{p}-\mathbf{c}_{r j}\right)^{T} \mathbf{E}^{T} \mathbf{u}_{1 o}+\mathbf{r}_{1 o}^{T} \mathbf{E}^{T} \mathbf{u}_{1 o}\right) \tau_{1 o} \\
\leq \frac{n_{r j 2}}{d_{r j 2}}\left(c_{2 j} \mathbf{u}_{1 o}^{T} \mathbf{E}^{T} \mathbf{u}_{1 j}+c_{1 o} \mathbf{u}_{1 j}^{T} \mathbf{E}^{T} \mathbf{u}_{2 j}+c_{1 j} \mathbf{u}_{2 j}^{T} \mathbf{E}^{T} \mathbf{u}_{1 o}\right) \tau_{1 o} \\
\left\|\left(\left(\mathbf{p}-\mathbf{c}_{r j}\right)^{T}-\frac{n_{r j 2}}{d_{r j 2}}\left(c_{2 j} \mathbf{u}_{1 j}^{T}-c_{1 j} \mathbf{u}_{2 j}^{T}\right)\right) \mathbf{E}^{T} 1+\frac{n_{r j 2}}{d_{r j 2}}\left(\mathbf{u}_{1 j}^{T} \mathbf{E}^{T} \mathbf{u}_{2 j}\right)\right\|
\end{gathered}
$$

where $n_{r j 1}, d_{r j 1}, n_{r j 2}$ and $n_{r j 2}$ are constants expressed as:

$$
\begin{aligned}
n_{r j 1} & =\left(\mathbf{p}-\mathbf{c}_{r j}\right)^{T} \mathbf{E}^{T} \mathbf{u}_{1 o}+\mathbf{r}_{1 o}^{T} \mathbf{E}^{T} \mathbf{u}_{1 o} \\
n_{r j 2} & =\left(\mathbf{p}-\mathbf{c}_{r j}\right)^{T} \mathbf{E}^{T} \mathbf{u}_{2 o}+\mathbf{r}_{2 o}^{T} \mathbf{E}^{T} \mathbf{u}_{2 o} \\
d_{r j 1} & =c_{1 o} \mathbf{u}_{2 j}^{T} \mathbf{E}^{T} \mathbf{u}_{1 j}^{T}+c_{1 j} \mathbf{u}_{1 o}^{T} \mathbf{E}^{T} \mathbf{u}_{2 j}^{T}+c_{2 j} \mathbf{u}_{1 j}^{T} \mathbf{E}^{T} \mathbf{u}_{1 o}^{T} \\
d_{r j 2} & =c_{2 o} \mathbf{u}_{2 j}^{T} \mathbf{E}^{T} \mathbf{u}_{1 j}^{T}+c_{2 j} \mathbf{u}_{1 j}^{T} \mathbf{E}^{T} \mathbf{u}_{2 o}^{T}+c_{1 j} \mathbf{u}_{2 o}^{T} \mathbf{E}^{T} \mathbf{u}_{2 j}^{T}
\end{aligned}
$$

Equations (20) and (21) represent the SE constraint associated with $\mathscr{M}_{j}$ at the contact point $C_{r j}$ in the form of hyperplanes [10]. Similarly the SE constraints generated at the contact point $C_{l j}$ will also form $C_{n-m_{j}}^{m_{o}}$ number of hyperplanes in the wrench space by substituting Eqns. (17) and (18) separately in Eqn. (14). Following the approach presented in [6], these constraints in the wrench space can be directly used to determine the facets of the AWS associated with the SE of the MBs.

The Capacity Margin $[12,13]$ determines if a given pose is wrench feasible using the facets of the AWS and the vertices of the Required Wrench Set (RWS). It is a measure of the robustness of the equilibrium of the robot, expressed by $\mu$,

$$
\mu=\min \left(\min s_{d, l}\right),
$$

where $s_{d, l}$ is the signed distance from $d$ th vertex of the RWS to the $l$ th face of the AWS. $\mu$ is positive as long as all the vertices of RWS are inscribed by $\mathscr{A}$, i.e. RWS can be counter balanced by the wrenches generated by the cables while respecting all the SE constraints.

\section{KINEMATIC MODELING AND AVAILABLE TWIST SET}

This section presents the first order kinematic model of the MCDPR under study. For classical CDPRs, twist of the MP can be expressed as [14]:

$$
\left[\begin{array}{l}
\mathbf{A}_{1} \\
\mathbf{A}_{2}
\end{array}\right]{ }^{0}{ }_{\mathbf{t}_{P}^{\text {cables }}}=\left[\begin{array}{l}
\mathbf{i}_{1} \\
\dot{\mathbf{I}}_{2}
\end{array}\right],
$$

where $\mathbf{A}_{j}$ is a $(2 \times n)$ parallel Jacobian matrix, containing the actuation wrenches due to the cables attached to $\mathscr{M}_{j}$ on the MP expressed as:

$$
\mathbf{A}_{j}=\left[\begin{array}{ll}
\mathbf{u}_{1 j}^{T} & \mathbf{r}_{1 j}^{T} \mathbf{E}^{T} \mathbf{u}_{1 j} \\
\mathbf{u}_{2 j}^{T} & \mathbf{r}_{2 j}^{T} \mathbf{E}^{T} \mathbf{u}_{2 j}
\end{array}\right],
$$

The twist ${ }^{0} \mathbf{t}_{P}^{\text {cables }}=\left[\begin{array}{ll}\dot{\mathbf{p}} & \omega\end{array}\right]^{T}$ is composed of the platform linear velocity vector $\dot{\mathbf{p}}=\left[\dot{p}_{x}, \dot{p}_{y}\right]^{T}$ and angular velocity $\omega$, all expressed in the base frame $\mathscr{F}_{0} .{ }^{0} \mathbf{t}_{P}^{\text {cables }}$ denotes the MP twist due to the motion of the cables expressed in $\mathscr{F}_{0} . \mathbf{i}_{j}=\left[\begin{array}{ll}i_{1 j} & i_{2 j}\end{array}\right]^{T}$ is a two-dimensional cable velocity vector of the cables attached to $\mathscr{M}_{j}$. It should be noted that the kinematic model expressed in Eqn. (24) has one degree of actuation redundancy, i.e. one actuator more than strictly necessary to control all DoF of the moving platform.

For MCDPRs, the twist generated onto the MP is due to both the cables and the MBs. Therefore the twist ${ }^{0} \mathbf{t}_{P}^{j}$ of the MP due to the $j$ th limb can be expressed as:

$$
{ }^{0} \mathbf{t}_{P}^{j}=\mathbf{J}_{b} \dot{\rho}_{j}+{ }^{0} \mathbf{R}_{b j}{ }^{b j} \mathbf{t}_{P}^{\text {cables }_{j}}
$$

where $\mathbf{J}_{b}=\left[\begin{array}{lll}1 & 0 & 0\end{array}\right]^{T} \cdot \dot{\rho}_{j}$ represents the velocity of $\mathscr{M}_{j} \cdot{ }^{b j_{j}} \mathbf{t}_{P}^{\text {cables }}{ }^{{ }^{2}}$ is the twist generated by the cables attached to $\mathscr{M}_{j}$ expressed in $\mathscr{F}_{b j} .{ }^{0} \mathbf{R}_{b j}$ is the rotation matrix between frames $\mathscr{F}_{b j}$ and $\mathscr{F}_{0}$. Upon multiplication of Eqn. (26) with $\mathbf{A}_{j}$ :

$$
\mathbf{A}_{j}{ }^{0} \mathbf{t}_{P}^{j}=\mathbf{A}_{j} \mathbf{J}_{b} \dot{\rho}_{j}+\mathbf{A}_{j}{ }^{0} \mathbf{R}_{b j}{ }^{b j} \mathbf{t}_{P}^{\text {cables }_{j}}
$$

where $\mathbf{A}_{j}{ }^{0} \mathbf{R}_{b j}{ }^{{ }^{b j}} \mathbf{t}_{P}^{\text {cables }_{j}}$ corresponds to $\dot{\mathbf{I}}_{1}$ (see Eqn. (24)). Thus Eqn. (27) can be expressed as:

$$
\mathbf{A}_{j}{ }^{0} \mathbf{t}_{P}^{j}=\mathbf{A}_{j} \mathbf{J}_{b} \dot{\rho}_{j}+\mathbf{i}_{j}
$$


The twists generated by both limbs is equal to the twist of the MP $\mathbf{t}_{P}$, namely,

$$
{ }^{0} \mathbf{t}_{P}^{1}={ }^{0} \mathbf{t}_{P}^{2}=\mathbf{t}_{P}
$$

Thus, in terms of both the limbs, MP twist of the planar FASTKIT can be expressed as:

$$
\left[\begin{array}{l}
\mathbf{A}_{1} \\
\mathbf{A}_{2}
\end{array}\right] \mathbf{t}_{P}=\left[\begin{array}{cc}
\mathbf{A}_{1} \mathbf{J}_{b} & 0 \\
0 & \mathbf{A}_{2} \mathbf{J}_{b}
\end{array}\right] \dot{\mathbf{q}}_{b}+\mathbf{i}
$$

where $\dot{\mathbf{q}}_{b}=\left[\begin{array}{ll}\dot{\rho}_{1} & \dot{\rho}_{2}\end{array}\right]^{T}$ and $\mathbf{i}=\left[\begin{array}{ll}\dot{\mathbf{i}}_{1} & \dot{\mathbf{l}}_{2}\end{array}\right]^{T}$. As the passive mobile base is fixed during the task mode, thus $\dot{\rho}_{1}=0$.

$$
\mathbf{A} \mathbf{t}_{P}=\mathbf{B}_{b} \dot{\mathbf{q}}_{b}+\mathbf{i}
$$

$$
\mathbf{A t} \mathbf{t}_{P}=\mathbf{B} \dot{\mathbf{q}}
$$

where $\mathbf{B}=\left[\begin{array}{ll}\mathbf{B}_{b} & \mathbf{I}_{m}\end{array}\right]$ is a $(4 \times 6)$ matrix while $\dot{\mathbf{q}}=\left[\begin{array}{ll}\dot{\mathbf{q}}_{b} & \mathbf{i}\end{array}\right]^{T}$ is a six dimensional vector containing all the joint velocities. From Eqn. (32), it can be observed that if $\mathbf{B}$ has rank four, the MCDPR under study has one degree of kinematic redundancy due to the motion of the active mobile base.

For trajectory planning, it is necessary to determine the set of twist feasible poses of the MP known as Available Twist Set (ATS). In [7], authors propose the methodology to determine ATS of MCDPRs using the first order kinematic model of the latter. According to [7], similar to AWS the ATS of MCDPRs is a convex polytope. ATS strictly depends on the robot configuration and the joint velocity limits, i.e. velocity limits for the cables and MBs. Similar to Eqn. (23), the Capacity Margin index is used to determine if the given pose is twist feasible by utilizing the facets of ATS and the vertices of the Required Twist Set (RTS), expressed by $v$ :

$$
v=\min \left(\min e_{d, l}\right)
$$

where $e_{d, l}$ is the signed distance from $d$ th vertex of the Required Twist Set (RTS) to the $l$ th face of the ATS. $v$ is positive as long as the platform have the ability to generate the RTS.

\section{OPTIMUM KINEMATIC REDUNDANCY SCHEME}

This section deals with a methodology that aims to determine the best $\rho_{2}$ for the adopted pick-and-place trajectory shown in Fig. 3. The proposed methodology is highlighted by defining a wrench quality criterion and formulation of a bi-objective optimization problem.

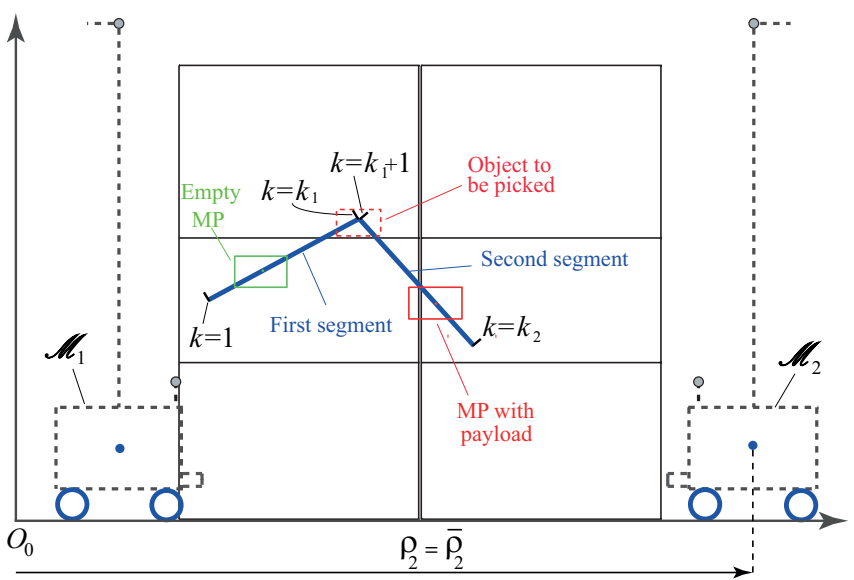

FIGURE 3. ADOPTED PICK AND PLACE PATH

\subsection{Objective Function}

The adopted path is composed of two segments, i.e. picking segment discretized into $k_{1}$ points and placing segment discretized into $k_{2}-k_{1}$ points shown in Fig. 3. Thus the complete path is discretized into $k_{2}$ points with each point denoted by $k$ such that, $k=1, \ldots, k_{1}, k_{1}+1, \ldots, k_{2}$. Let $t_{1}\left(m_{1}\right.$, resp.) and $t_{2}\left(m_{2}\right.$, resp.) be the trajectory time (total moving mass, resp.) for the first and second segments of pick-and-place operation. It should be noted that the adopted trajectory is linear and does not require any rotational motion of the MP.

For a fast pick-and-place trajectory operation, it makes sense to minimize the total trajectory time, thus the first objective function can be expressed as:

$$
\text { Minimize } f_{1}=t_{1}+t_{2}
$$

The second objective function aims at maximizing the robustness index (see Eqn. (23)) of the MP for the complete path, thus the second objective function can be expressed as:

$$
\operatorname{Maximize} f_{2}=\frac{m_{1}}{k_{1}} \sum_{k=1}^{k_{1}} \mu_{k}+\frac{m_{2}}{k_{2}-k_{1}} \sum_{k=k_{1}+1}^{k_{2}} \mu_{k}
$$

$\mu_{k}$ being the capacity margin defined in Eqn. (23) and assessed at the $k$ th point of the piecewise path. Average robustness index for each segment of the path in Eqn. (35) takes into account the mass of the MP as well to have an equal ratio between the indices as it tends to decrease with the increase in the MP mass and vice versa.

\subsection{Decision Variables}

The decision variable vector of the optimization problem contains the trajectory time of both the segments $\left(t_{1}, t_{2}\right)$ and re- 
dundancy planning scheme. Let $\beta_{k}$ denote the redundancy planning scheme containing the position of $\mathscr{M}_{2}$ for each $k$ th discretized point such that:

$$
\beta=\left[\begin{array}{llllll}
\rho_{21} & \rho_{22} & \ldots & \rho_{2 k_{1}} & \ldots & \rho_{2 k_{2}}
\end{array}\right]^{T}
$$

with $\underline{\rho}_{2} \leq \beta_{k} \leq \bar{\rho}_{2}, k=1, \ldots, k_{1}, k_{1}+1, \ldots, k_{2} . \underline{\rho}_{2}$ and $\bar{\rho}_{2}$ denote the lower and upper bounds on $\rho_{2}$.

\subsection{Constraints}

Six types of constraints are taken into account in the optimization problem:

1. As the passive mobile base is fixed, thus the velocity of $\mathscr{M}_{1}$ is zero,

$$
\dot{\rho}_{1}=0
$$

2. The MP pose must be capable of generating the RWS and RTS along the trajectory. RWS depends on mass and the required acceleration from the pick-and-place trajectory the MP. RTS is equal to the required twist of the MP. Thus for each $k$ th trajectory point, the indices $\mu_{k}$ and $v_{k}$ from Eqns. (23) and (33) must be positive, namely,

$$
\begin{aligned}
& \mu_{k} \geq 0, \\
& v_{k} \geq 0 .
\end{aligned}
$$

Equation (38a) ensures that the MP has the ability to generate the RWS while respecting the cable tension tension limits and the SE constraints associated with the MBs. Equation (38b) ensures that the MP can generate the RTS while respecting the joint velocity limits.

3. $\rho_{2}$ is bounded between its lower bound $\underline{\rho}_{2}$ and its upper bound $\bar{\rho}_{2}$ at each $k$ th trajectory point, namely,

$$
\underline{\rho}_{2} \leq \rho_{2 k} \leq \bar{\rho}_{2}
$$

4. $\dot{\rho}_{2}$ is also bounded between its lower bound $\dot{\rho}_{2}$ and its upper bound $\dot{\bar{\rho}}_{2}$ at each $k$ th trajectory point, namely,

$$
\underline{\dot{\rho}}_{2} \leq \dot{\rho}_{2 k} \leq \dot{\bar{\rho}}_{2}
$$

5. The first path starts with the MCDPR in undeployed configuration. Thus,

$$
\rho_{21}=\underline{\rho}_{2}
$$

6. The search for an optimal trajectory time of bounded as:

$$
\begin{aligned}
& 0 \leq t_{1} \leq \bar{t}_{1} \\
& 0 \leq t_{2} \leq \bar{t}_{2}
\end{aligned}
$$

\subsection{Formulation of the optimization problem}

In order to find the optimal kinematic redundancy scheme, the optimization problem formulated from Eqns. (34) to (42) is expressed as follows:

$$
\begin{aligned}
& \text { Minimize } \quad f_{1}(\mathbf{x})=t_{1}+t_{2} \\
& \text { Maximize } \quad f_{2}(\mathbf{x})=\frac{m_{1}}{k_{1}} \sum_{k=1}^{k_{1}} \mu_{k}+\frac{m_{2}}{k_{2}-k_{1}} \sum_{k=k_{1}+1}^{k_{2}} \mu_{k} \\
& \text { over } \mathbf{x}=\left[\begin{array}{lll}
\beta & t_{1} & t_{2}
\end{array}\right] \\
& \text { subject to } \quad \dot{\rho}_{1}=0 \\
& \rho_{21}=\underline{\rho}_{2} \\
& \underline{\rho}_{2} \leq \rho_{2 k} \leq \bar{\rho}_{2} \\
& \dot{\rho}_{2} \leq \dot{\rho}_{2 k} \leq \bar{\rho}_{2} \\
& \mu_{k} \geq 0 \\
& v_{k} \geq 0 \\
& 0 \leq t_{1} \leq \bar{t}_{1} \\
& 0 \leq t_{2} \leq \bar{t}_{2} \\
& k=1, \ldots, k_{1}, k_{1}+1, \ldots, k_{2}
\end{aligned}
$$

The optimization problem formulated in Eqn. (43) aims at finding the the optimum trajectory time of each segment $\left(t_{1}\right.$ and $\left.t_{2}\right)$ and the corresponding optimum kinematic redundancy scheme $(\beta)$ that minimize the total trajectory time $\left(f_{1}\right)$ and maximize the criterion $f_{2}$ defined in Eqn. (35) while respecting the set of constraints. The foregoing optimization problem is solved for a case study in the following section.

\section{CASE STUDY}

All the parameters required to acquire and analyze the results for the optimization problem defined in Eqn. (43) are presented in section 6.1. The results are analyzed in section 6.2.

\subsection{Parameters}

Both segments of the pick-and-place path are discretized into 50 such that $k_{2}=2 k_{1}=100$. The maximum trajectory time of each segment is defined as:

$$
\bar{t}_{1}=\bar{t}_{2}=10 \mathrm{~s}
$$


The mass of the MP is taken as:

$$
m_{1}=1 \mathrm{~kg}, \quad m_{2}=2.5 \mathrm{~kg}
$$

The weight vector of the MBs is defined as,

$$
\mathbf{w}_{g j}=m_{m b j}\left[\begin{array}{c}
0 \\
-g
\end{array}\right] \mathrm{N}, \quad j=\{1,2\}
$$

where $g=9.81 \mathrm{~m} . \mathrm{s}^{-2}$ represents the acceleration due to gravity. $m_{m b 1}=m_{m b 2}=150 \mathrm{~kg}$ represent the mass of $\mathscr{M}_{1}$ and $\mathscr{M}_{2}$. The joint velocity limits i.e. velocity limits of the cables and MBs are defined as:

$$
\begin{gathered}
\underline{\rho}_{2}=-0.2 \mathrm{~m} \cdot \mathrm{s}^{-1}, \quad \dot{\bar{\rho}}_{2}=0.2 \mathrm{~m} \cdot \mathrm{s}^{-1} \\
-2 \mathrm{~m} \cdot \mathrm{s}^{-1} \leq i_{i j} \leq 2 \mathrm{~m} \cdot \mathrm{s}^{-1}, \quad i=\{1,2\}, j=\{1,2\}
\end{gathered}
$$

The cable tension limits are defined as:

$$
\underline{\tau}_{i j}=0, \quad \bar{\tau}_{i j}=45 \mathrm{~N}, \quad i=\{1,2\}, j=\{1,2\}
$$

The bounds on the position of $\mathscr{M}_{2}$ are defined as:

$$
\underline{\rho}_{2}=1.1 \mathrm{~m}, \quad \bar{\rho}_{2}=4 \mathrm{~m}
$$

which means that the robot can be deployed up to maximum $\bar{\rho}_{2}-\underline{\rho}_{2}=2.9 \mathrm{~m}$.

\subsection{Result Analysis}

It is noteworthy that the only decision variables that are considered to solve the proposed optimization problem are $t_{1}$ and $t_{2}$, respectively. The vector $\beta$ is searched at each iteration and is obtained in such a way that it minimizes the objective function $f_{1}$, maximizes the objective function $f_{2}$ and leads to a continuous solution for $\rho_{2}$ along the trajectory. The feasible solutions in decision space is illustrated in Fig. 4. On the left side of the transition curve, there exists no solution between $t_{1}$ and $t_{2}$ that respect all the constraints defined in Eqn. (43) referred to as unfeasible time set. On the right side of the transition curve, each combination between $t_{1}$ and $t_{2}$ fulfills all the constraints of the proposed optimization problem (43) referred to as feasible time set.

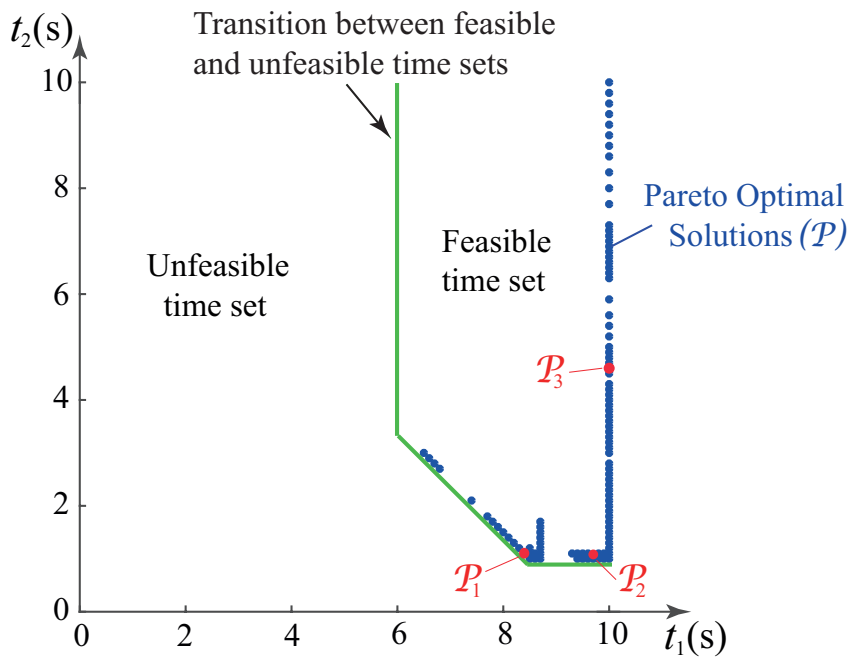

FIGURE 4. FEASIBLE AND UNFEASIBLE TIME SETS

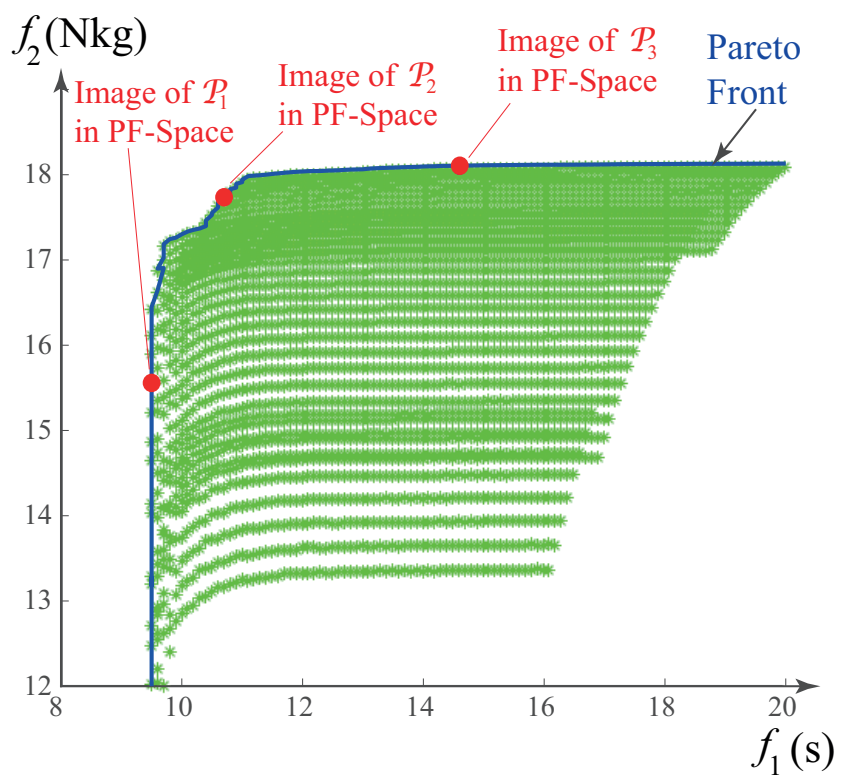

FIGURE 5. ALL FEASIBLE SOLUTIONS (GREEN), PARETO FRONT (BLUE) AND THREE PARETO-OPTIMAL SOLUTIONS (RED) IN THE PERFORMANCE FUNCTION SPACE (PF-SPACE)

The optimization problem in Eqn. (43) has more than one optimal solution. These optimal solutions are defined as Paretooptimal solutions, which cannot be dominated by any other feasible solution $[15,16]$. The set of all Pareto-optimal solutions is called as Pareto optimal set, denoted by $\mathscr{P}$, illustrated in Fig. 4. The Pareto-optimal solutions lie on a boundary in the Perfor- 


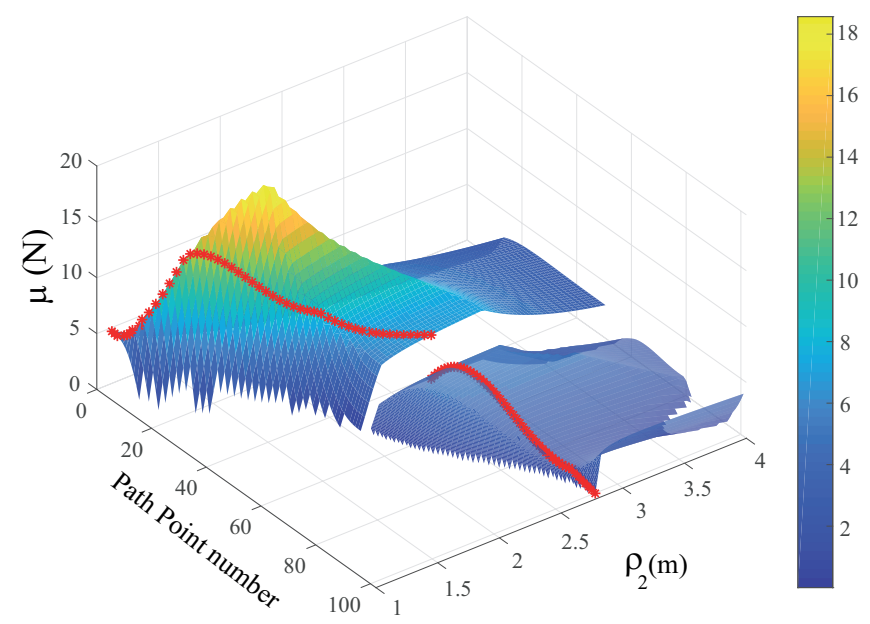

FIGURE 6. $\quad \mu$ AS A FUNCTION OF PATH POINT NUMBER AND $\rho_{2}$ FOR PARETO-OPTIMAL SOLUTION $\mathscr{P}_{1}$, i.e. $t_{1}=8.4 \mathrm{~s}$ AND $t_{2}=$ 1.1s. THE RED CURVE HIGHLIGHTS OPTIMUM REDUNDANCY SCHEME

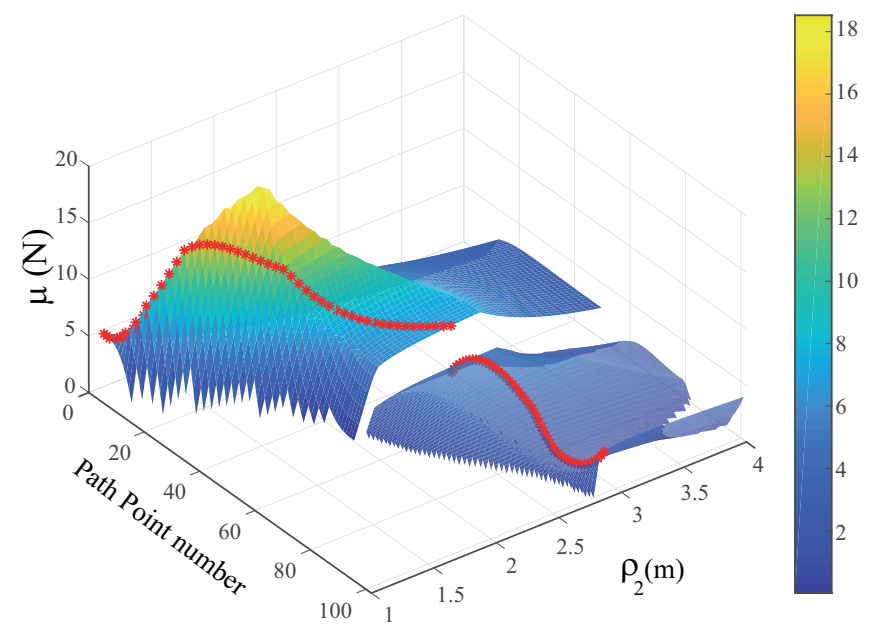

FIGURE 7. $\mu$ AS A FUNCTION OF PATH POINT NUMBER AND $\rho_{2}$ FOR PARETO-OPTIMAL SOLUTION $\mathscr{P}_{2}$, i.e. $t_{1}=9.7 \mathrm{~s}$ AND $t_{2}=$ 1.1s. THE RED CURVE HIGHLIGHTS OPTIMUM REDUNDANCY SCHEME

mance Function Space (PF-Space) between the two objective functions called Pareto front [17] shown in Fig. 5. It can be observed in Fig. 4 that most of the Pareto-optimal solution requires the largest $t_{1}$. This behavior can be explained from the definition of the adopted pick-and-place path.

During the first segment of the path (see Fig. 3), the platform height is increased that reduces the AWS along $y_{0}$. The RWS due to the acceleration of the MP tends to decrease with the increase in $t_{1}$, which results in higher $f_{2}$ along the first segment. The sit-

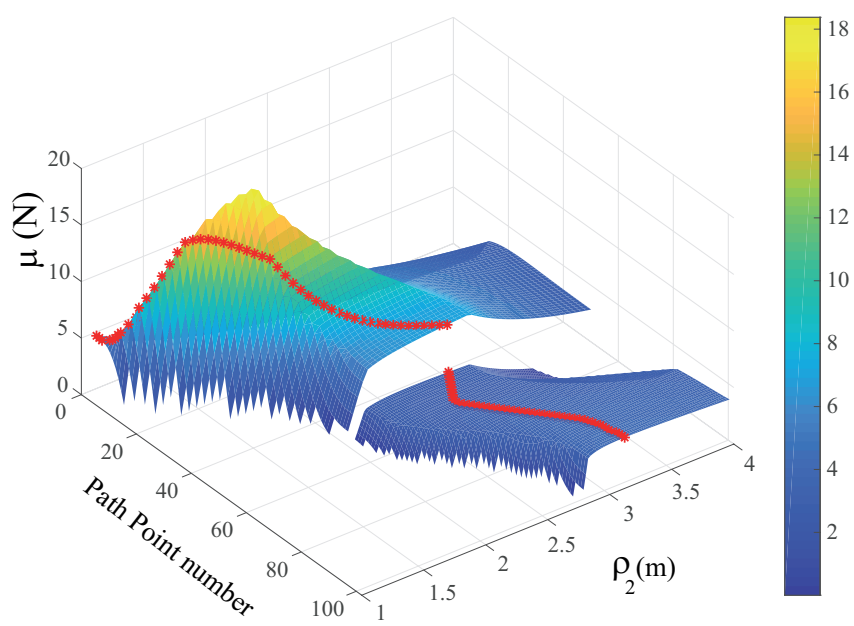

FIGURE 8. $\mu$ AS A FUNCTION OF PATH POINT NUMBER AND $\rho_{2}$ FOR PARETO-OPTIMAL SOLUTION $\mathscr{P}_{3}$, i.e, $t_{1}=10 \mathrm{~s}$ AND $t_{2}=$ 4.6s. THE RED CURVE HIGHLIGHTS OPTIMUM REDUNDANCY SCHEME

uation is opposite for the second segment of the adopted path as AWS tends to increase along $y_{0}$ with the decrease in the platform height. The similar phenomenon can also be observed in Fig. 5 through transition curve where the minimum time that is required to find a feasible solution for first segment is equal to $6 \mathrm{~s}$ while it is equal to $1 \mathrm{~s}$ only for the second segment. It should be noted that at $t_{1}=\bar{t}_{1}$, changing $t_{2}$ does not produce any prominent change in $f_{2}$ (see Figs. 4 and 5). Thus, it can be concluded that the transition curve i.e. the feasible and unfeasible time set is highly dependent of the adopted path.

To illustrate an optimal redundancy scheme, three different Pareto-optimal solutions, i.e. $\mathscr{P}_{1}, \mathscr{P}_{2}$ and $\mathscr{P}_{3}$, have been selected in Figs. 4 and 5. Figures 6, 7 and 8 show the index $\mu$ as a function of the path point number and $\rho_{2}$ for the chosen Pareto-optimal solutions $\mathscr{P}_{1}, \mathscr{P}_{2}$ and $\mathscr{P}_{3}$ known as efficiency maps [18]. The red curve in the efficiency maps highlights the optimum redundancy scheme. The white areas in the map corresponds to the region where the constraints of the proposed optimization problem are not satisfied. It can be observed that there is a sudden change in $\mu$ at $k=\frac{k_{2}}{2}$. It is due to the fact that the weight of the MP is changed at the end of the first segment of pick-and-place operation. Due to this following reason, the mass of the MP is taken into account in the definition of the second objective function $f_{2}$, which is expressed in Eqn. (35).

The simulation videos for corresponding pick-and-place operation for all the three Pareto-optimal solutions $\mathscr{P}_{1}, \mathscr{P}_{2}$ and $\mathscr{P}_{3}$ can be downloaded at ${ }^{2}$.

\footnotetext{
${ }^{2}$ https://youtu.be/fpdlEnYbP7M
} 


\section{CONCLUSION}

In this paper, a methodology to determine the optimal kinematic redundancy scheme of Planar Mobile Cable Driven Parallel Robots (PMCDPRs) with one degree of kinematic redundancy for fast pick-and-place operations is described. First, the Static equilibrium (SE) constraints of PMCDPRs associated with the Mobile Bases (MBs) are formulated that are required to fully characterize the Available Wrench Set (AWS) of the latter. Then, a bi-objective optimization problem that corresponds to minimization of the total trajectory time and maximization of the robot average robustness index throughout the trajectory is formulated in order to determine the optimum kinematic redundancy scheme. A case study of a PMCDPR composed of two $\mathrm{MBs}$, four cables and a three degree-of-freedom (DoF) moving platform is considered. Future work will deal with the experimental validation and the extension of the proposed methodology to spatial Mobile Cable Driven Parallel Robots (MCDPRs) with higher degrees of kinematic redundancy.

\section{ACKNOWLEDGMENT}

This research work is part of the European Project ECHORD++ "FASTKIT" dealing with the development of collaborative and Mobile Cable-Driven Parallel Robots for logistics. Ecole Centrale Nantes is also dutifully acknowledged for the financial support provided to the first author of the paper.

\section{REFERENCES}

[1] Kawamura, S., Kino, H., and Won, C., 2000. "High-speed manipulation by using parallel wire-driven robots". Robotica, 18(1), pp. 13-21.

[2] Albus, J., Bostelman, R., and Dagalakis, N., 1993. "The nist robocrane". Journal of Field Robotics, 10(5), pp. 709724.

[3] Lambert, C., Nahon, M., and Chalmers, D., 2007. "Implementation of an aerostat positioning system with cable control". IEEE/ASME Transactions on Mechatronics, 12(1), pp. 32-40.

[4] Gagliardini, L., Caro, S., Gouttefarde, M., and Girin, A., 2016. "Discrete reconfiguration planning for cable-driven parallel robots". Mechanism and Machine Theory, 100, pp. 313-337.

[5] Rasheed, T., Long, P., Marquez-Gamez, D., and Caro, S., 2018. "Tension distribution algorithm for planar mobile cable-driven parallel robots". In Cable-Driven Parallel Robots. Springer, pp. 268-279.

[6] Rasheed, T., Long, P., Gamez, D. M., and Caro, S. "Available wrench set for planar mobile cable-driven parallel robots". In 2018 IEEE International Conference on Robotics and Automation (ICRA).
[7] Rasheed, T., Long, P., Gamez, D. M., and Caro, S. "Kinematic modeling and twist feasibility of mobile cable-driven parallel robots". In Advances in Robot Kinematics 2018.

[8] Kawamura, S., and Ito, K., 1993. "A new type of master robot for teleoperation using a radial wire drive system". In Proceedings IEEE/RSJ Intelligent Robots and System (IROS) 1993, Vol. 1, IEEE, pp. 55-60.

[9] Hiller, M., Fang, S., Mielczarek, S., Verhoeven, R., and Franitza, D. "Design, analysis and realization of tendonbased parallel manipulators". Mechanism and Machine Theory, 40(4), pp. 429-445, 2005.

[10] Gouttefarde, M., and Krut, S., 2010. "Characterization of parallel manipulator available wrench set facets". In $A d$ vances in robot kinematics: motion in man and machine. Springer, pp. 475-482.

[11] Bouchard, S., Gosselin, C., and Moore, B., 2010. "On the ability of a cable-driven robot to generate a prescribed set of wrenches". Journal of Mechanisms and Robotics, 2(1), p. 011010.

[12] Guay, F., Cardou, P., Cruz-Ruiz, A. L., and Caro, S., 2014. "Measuring how well a structure supports varying external wrenches". In New Advances in Mechanisms, Transmissions and Applications. Springer, pp. 385-392.

[13] Ruiz, A. L. C., Caro, S., Cardou, P., and Guay, F., 2015. "Arachnis: Analysis of robots actuated by cables with handy and neat interface software". In Cable-Driven Parallel Robots. Springer, pp. 293-305.

[14] Roberts, R. G., Graham, T., and Lippitt, T., 1998. “On the inverse kinematics, statics, and fault tolerance of cablesuspended robots". Journal of Field Robotics, 15(10), pp. 581-597.

[15] Bui, L. T., Abbass, H. A., Barlow, M., and Bender, A., 2012. "Robustness against the decision-maker's attitude to risk in problems with conflicting objectives". IEEE Transactions on Evolutionary Computation, 16(1), pp. 1-19.

[16] Li, X., and Wong, H.-S., 2009. "Logic optimality for multiobjective optimization". Applied Mathematics and Computation, 215(8), pp. 3045-3056.

[17] Wang, W., Caro, S., Bennis, F., Soto, R., and Crawford, B., 2015. "Multi-objective robust optimization using a postoptimality sensitivity analysis technique: application to a wind turbine design”. Journal of Mechanical Design, 137(1), p. 011403.

[18] Caro, S., Garnier, S., Furet, B., Klimchik, A., and Pashkevich, A., 2014. "Workpiece placement optimization for machining operations with industrial robots". In Advanced Intelligent Mechatronics (AIM), 2014, IEEE, pp. 1716-1721. 